\title{
Usar ou não usar rubricas? Um olhar para as práticas avaliativas a partir dos desempenhos discentes
}

\author{
Use or not use rubrics? A look at assessment practices \\ from student performances
¿Usar o no rúbricas? Una mirada hacia las prácticas evaluativas a partir de los rendimientos estudiantiles

\author{
Leandro Blass (leandroblass@unipampa.edu.br) \\ Universidade Federal do Pampa-UNIPAMPA.
}

\section{Valesca Brasil Irala (valescairala@unipampa.edu.br)}

Universidade Federal do Pampa- UNIPAMPA.

Resumo: O objetivo do artigo é relatar o desenvolvimento das práticas avaliativas adotadas como estratégias docentes ao longo de um conjunto de seis semestres letivos, nos quais três deles foram caracterizados pelo uso de rubricas e três deles não, em uma disciplina de Cálculo Numérico ministrada pelo mesmo professor a diferentes cursos de graduação. A metodologia adotada é mista (quanti-quali), com predominância do viés quantitativo e foco na intervenção pedagógica. Ao todo, foram analisados desempenhos de 305 alunos. Os dados foram tratados por meio da Estatística Descritiva e foi realizado o teste não-paramétrico de Kruskal-Wallis, no qual se constatou diferença nos desempenhos entre os semestres com e sem adoção de rubricas. O pos hoc identificou que apenas o semestre 2017.2 (sem rubricas) não diferiu do semestre 2019.1 (com rubricas). Conclui-se, de forma geral, que os resultados se mostraram favoráveis à adoção de rubricas na avaliação discente.

Palavras-chave: Rubrica; Avaliação; Ensino Superior.

\begin{abstract}
The main aim of this study is to report the development of the assessment practices adopted as teaching strategies over a set of six academic semesters, in which three of them were characterized by the use of rubrics and three of them not, in a Numerical Analysis course taught by the same teacher to different undergraduate courses. The methodology adopted is mixed (quanti-quali), with a predominance of quantitative bias and focus on pedagogical interventio. In all, 305 students' performances were analyzed. The data were treated using Descriptive Statistics and the Kruskal-Wallis non-parametric test was performed, in which a difference in performance was found between the semesters with and without the adoption of rubrics. The pos hoc identified that only the 2017.2 semester (without headings) did not differ from the 2019.1 semester (with headings). It is concluded, in general, that the results were favorable to the adoption of rubrics in the student assessment.
\end{abstract}


Keywords: Rubric; Assessment; Higher Education.

Resumen: El objetivo del artículo es reportar el desarrollo de las prácticas evaluativas adoptadas como estrategia docente a lo largo de un conjunto de seis semestres académicos, en los cuales tres de ellos se caracterizaron por el uso de rúbricas y tres de ellos no, en una asignatura de Cálculo Numérico impartida por el mismo docente para diferentes cursos de pregrado. La metodología adoptada es mixta (cuanti-cuali), con predominio del sesgo cuantitativo y foco en la intervención pedagógica. En total, se analizaron las actuaciones de 305 estudiantes. Los datos se trataron mediante Estadística Descriptiva y se realizó la prueba no paramétrica de Kruskal-Wallis, en la que se encontró diferencia de desempeño entre los semestres con y sin adopción de rúbricas. El pos hoc identificó que solo el semestre 2017.2 (sin rúbricas) no difirió del semestre 2019.1 (con rúbricas). Se concluye, en general, que los resultados fueron favorables a la adopción de rúbricas en la evaluación del alumnado.

Palabras-clave: Rúbrica; Evaluación; Educación Superior.

1.

\section{INTRODUÇÃO}

A disciplina de Cálculo Numérico possui um contexto muito vasto de possíveis aplicações (COBO, 2008). Como forma de contemplar a exploração de aplicações de interesse de estudantes de diferentes cursos de graduação, em ofertas contínuas pelo mesmo professor na disciplina para turmas multicurso (que envolve tanto discentes de diversas Engenharias quanto de Licenciaturas), na Universidade Federal do Pampa (UNIPAMPA), no campus Bagé, surgiu inicialmente a proposta de avaliação discente via formação de grupos voltados à investigação sobre aplicações específicas de interesse dos estudantes, culminando com a socialização dos resultados através de um seminário para apresentação oral e entrega de documento escrito da pesquisa efetivada.

A presente pesquisa foi motivada a partir de reflexões advindas da prática docente em suas primeiras experiências frente à proposta avaliativa citada, tais como: qual a maneira mais adequada para acompanhar o processo dos alunos até a culminância do seminário? Como explicitar os critérios, as habilidades e formas de organização esperados? Esses e outros questionamentos pautaram mudanças avaliativas na disciplina de Cálculo Numérico em um campus universitário de uma universidade pública localizada no sul do Brasil. A opção por 
realizá-la nesta disciplina se deve ao fato de que o docente envolvido na implementação das rubricas tem recorrentemente sido o responsável por Cálculo Numérico no campus em que a pesquisa foi realizada e efetuado regularmente pesquisas de intervenção pedagógica vinculadas a esse componente (BLASS; IRALA, 2020a; 2020b; BLASS, GOERGEN, IRALA, 2020), no âmbito do Grupo de Pesquisa sobre Aprendizagens, Metodologias e Avaliação - GAMA (cadastrado no Diretório de Grupos de Pesquisa do CNPq).

Neste trabalho em específico, a pesquisa enfatiza o uso de rubricas (BROOKHART, 2013; BOSTON, 2002; HOWELL, 2014) para o acompanhamento das tarefas desenvolvidas pelos alunos no processo de investigação, tendo como culminância a realização dos seminários, pois o docente identificou que nos primeiros semestres de sua experiência docente existia um envolvimento muito pontual ou até mesmo nulo por parte de alguns discentes no interior dos grupos. Diante dessa realidade, as rubricas surgiram como estratégia para integrar os estudantes, padronizar os critérios, imprimir maior transparência ao processo e efetivo acompanhamento de todas as etapas previstas, até a sua finalização.

A rubrica pode ser definida aqui como um artefato avaliativo que guia o aluno, tendo por base elementos observáveis (MARCHETI, 2020), que, de antemão, permite que sejam conhecidos os critérios e as pontuações previamente determinados pelo professor, almejando o esforço discente para melhorar a aprendizagem diante da proposta avaliativa a ser realizada (OAKLEAF, 2009). I Esse conjunto de critérios deve ser coerente e bem descrito, para que o aluno tenha o real entendimento a respeito do seu trabalho em cada nível de desempenho (BROOKHART, 2013).

É mais indicado, para atender os propósitos e realidades específicas de cada contexto, que o próprio professor elabore a sua rubrica, em vez de utilizar rubricas prontas, especialmente as que se encontram na Internet, embora haja uma vasta oferta de opções, especialmente em língua inglesa (TIERNEY; SIMON, 2004). Em determinadas situações, também é salutar que as rubricas sejam construídas colaborativamente entre docentes e discentes, implicando em maior horizontalidade em todas as etapas do processo avaliativo.

O objetivo deste artigo é estabelecer uma comparação baseada no desempenho dos alunos em seis semestres letivos, três em que a rubrica não foi utilizada e três em que foi. Salienta-se que quando a rubrica ainda não fazia parte da prática avaliativa do professor, os 
alunos eram avaliados apenas em relação à apresentação oral e ao trabalho escrito; ou seja, sem considerar as etapas de construção, com foco apenas nos produtos derivados do processo investigativo em si. A introdução das rubricas, portanto, nesse contexto, visa contribuir para uma compreensão mais qualificada desse processo e sua contínua melhoria.

\section{A RUBRICA NA PRÁTICA AVALIATIVA}

Uma nova estratégia avaliativa se caracteriza pela sua possível aplicabilidade em contextos reais; por ser efetuada de maneira contínua; pela promoção da interação, pela clareza, por primar pela comunicação entre os pares; pela obtenção de informações sobre o processo e, como resultado, maior engajamento dos estudantes (SCALLON, 2004). O uso de rubricas na avaliação pode trazer elementos que evidenciam tais pressupostos, de maneira a qualificar à prática avaliativa, a qual é intrínseca a qualquer processo de ensinoaprendizagem, porém não tem se constituído, por diversos fatores, como um movimento fácil, devendo implicar em constante (re)posicionamento crítico e reflexivo de seus atores (VORPAGEL; UHMANN, 2018).

A rubrica tem sido tratada na prática avaliativa como uma ferramenta baseada em critérios de desempenho que explicitam as expectativas (ou mesmo visibilizar desempenhos possíveis, mas não desejáveis) em variados níveis de atuação por parte dos estudantes (STEVENS; LEVI, 2005). Ao explicitar diferentes níveis de desempenho, potencializam a visão antecipatória do docente a respeito da execução de uma determinada tarefa, bem como ajuda ao aluno a escalonar possíveis ações e/ou comportamentos observáveis que possam ser mais bem sucedidos, caso sejam executados. Tais escalas podem ser quantitativas e/ou qualitativas (GORDILLO; RODRÍGUEZ, 2010), mas a sua principal função é gerar nos estudantes um entendimento mais qualificado sobre os reais objetivos de aprendizagem por detrás de uma tarefa ou um conjunto de tarefas, direcionando-os para a qualificação dos resultados (COOPER; GARGAN, 2009).

Marín, Gutierrez e Londoño (2019) afirmam que a avaliação não pode estar limitada a um teste que visa apenas verificar a repetição de certos conteúdos, mas firmá-la especialmente com práticas alinhadas à avaliação formativa, superando definitivamente visões tecnicistas a respeito do ato de avaliar (LAMY; JACQUES; GALIETA, 2019). É preciso, em definitivo, 
avançar para a compreensão e intervenção sobre o processo de aprendizagem. Entende-se, neste trabalho, que as rubricas podem contribuir para essa mudança necessária de paradigmas no campo avaliativo, há tanto tempo anunciados na literatura da área.

As rubricas visam conduzir a uma avaliação transparente, participativa e mais inclusiva. Essas diretrizes são indispensáveis para que os procedimentos avaliativos contenham impactos positivos no processo de ensino-aprendizagem. Segundo Jácome (2013), deve ser esse o objetivo de qualquer avaliação em sala de aula, o que não acontece quando ela é restrita a testes focados em repetição (GARCIA, 2008). Para Stevens e Levi (2005), a avaliação com rubricas, seguida de uma qualificada orientação acadêmica, pode encorajar o aluno a desenvolver um pensamento crítico, aberto e independente para novos desafios.

As rubricas estão classificadas em holísticas e analíticas. As holísticas fornecem uma ideia global do desempenho dos alunos, pois ele é avaliado uma única vez em todas as tarefas, porém, ela não proporciona um feedback por cada atividade específica. Já as rubricas analíticas são capazes de dividir a avaliação de uma tarefa em etapas que o professor achar essencial, fazendo com que o aluno possa melhorar seu desempenho de forma situada, específica e gradual (BOSTON, 2002), dado o potencial de granularidade que apresenta. Nesta pesquisa, as rubricas criadas são de natureza holística, por serem consideradas, para o contexto analisado, as mais adequadas para otimizar o tempo destinado ao trabalho docente em comparação às analíticas e, do ponto de vista discente, para facilitar a sua compreensão, já que não é um dispositivo tão usual no contexto analisado.

As rubricas podem ajudar a responder algumas necessidades e problemáticas descritas por docentes na prática avaliativa diária, como relatam Stevens e Levi (2005): quando o professor tem dificuldades de expressar o que pretende que os alunos aprendam; quando há questionamentos sobre a consistência dos resultados de uma avaliação em comparação com a dos demais colegas de uma mesma turma; quando a avaliação é muita extensa e requer muito tempo de dedicação; quando surgem questionamentos sobre foco e consistência nos trabalhos realizados em aula por parte dos alunos; quando se revelam critérios diferentes de avaliação na correção dos trabalhos em cima de uma mesma tarefa (mesmo quando não há essa intencionalidade). Esses são, certamente, alguns aspectos recorrentes no campo da experiência avaliativa de professores em diferentes graus de atuação, contextos educacionais e níveis 
variados de aprofundamento em formações prévias no campo da docência universitária e no campo avaliativo.

A elaboração das rubricas pode variar de acordo com as características de avaliação específica; porém, é composta de um formato básico de quatro partes (STEVENS; LEVI, 2005): 1) descrição da tarefa; 2) uma escala ou nível de desempenho; 3) Os critérios de avaliação, as competências ou conhecimentos envolvidos ao realizar a tarefa; 4) os descritivos de cada nível.

É preciso destacar que as rubricas podem contribuir para a qualidade do feedback, que é um dos elementos centrais para que o processo avaliativo seja de fato qualificado e favoreça o avanço no nível de desempenho dos estudantes (MARÍN; GUTIERREZ, LONDOÑO, 2019). Oakleaf (2009) aponta que as rubricas fornecem informações sobre o que os alunos aprenderam e o que ainda precisam aprender. Outra vantagem é que o feedback gerado a partir de um Quadro de referência como a rubrica serve como ponto de partida para a criação da prática sistemática de autoavaliação por parte dos estudantes (TARAS, 2003).

A rubrica pode ser chamada de "orientadora" ou esclarecedora da forma que irá ocorrer a avaliação da aprendizagem (GATICA-LARA; URIBARREN-BERRUETA, 2013). Tal ideia é reforçada pela pesquisa de Nieminen e Pesonen (2020, p. 9), na qual estudaram a percepção de estudantes de graduação de Matemática identificados como "inclusos" (dislexia, espectro autista, etc.), a respeito do processo avaliativo mediado por rubricas, construído com base no Desenho Universal da Aprendizagem (DUA). Nas palavras dos autores: "os estudantes relataram que a rubrica e as práticas de autoavaliação tiveram um impacto positivo diante do aprender e estudar, permitindo definir metas e monitorar o próprio desempenho".

O trabalho de Carless (2019), realizado com quatro alunos de graduação, relatou que a estratégia de usar rubricas no processo de avaliar o ensino e aprendizagem é promissor e possibilita diretrizes de automonitoramento. Uma de suas vantagens, apontada pelo autor, é o engajamento dos alunos no ciclo de feedback, trazendo efeitos mais duradouros à aprendizagem. Em Raposo-Rivas e Martínez-Figueira (2014), a aplicação das rubricas ocorreu na disciplina Novas Tecnologias aplicadas à Educação Infantil, totalizando 71 alunos, distribuídos em 7 grupos experimentais (38 alunos) usando as rubricas e 6 grupos de controle (33 alunos), sem o uso das rubricas. Os resultados apontaram um melhor desempenho dos 
estudantes que foram avaliados com rubricas. De acordo com os alunos, a avaliação usando as rubricas possibilitou um processo mais justo, com objetivos claros, fortalecendo o desenvolvimento de competências voltadas à autoavaliação.

Já a pesquisa de Robles, Angulo e De la Serna (2014) teve objetivo de apresentar e discutir o uso de rubricas eletrônicas usadas para a avaliação formativa de alunos universitários de três instituições diferentes. Foram construídas rubricas focadas em conteúdos, no trabalho em equipe e na exposição de projetos. Os resultados da pesquisa apontaram para a melhoria na autorregulação da aprendizagem, tanto por meio da autoavaliação quanto da avaliação por pares, ambas mediadas pelas rubricas eletrônicas.

Em síntese, em diferentes contextos as rubricas têm sido evidenciadas como dispositivos úteis para promover melhorias no campo da avaliação discente. Uma compreensão situada e longitudinal como a realizada nesta pesquisa pode contribuir para a disseminação do uso dessa estratégia no ensino superior brasileiro, como já tem sido evidenciado há várias décadas em outros países, especialmente em contextos anglófonos.

\section{PROCEDIMENTOS METODOLÓGICOS E CONTEXTUALIZAÇÃO}

A tentativa de atrair o foco de interesse dos estudantes, bem como o estabelecimento de conexões para que o conhecimento de Cálculo Numérico seja voltado a cada área dos diversos cursos que ofertam a disciplina, foram os principais motores para a introdução de alternativas metodológicas e avaliativas no contexto educacional em que ela é ministrada (BLASS, IRALA, 2020b). Geralmente, os estudantes chegam para cursar Cálculo Numérico desacreditando que o seu conteúdo possa ser aplicado a suas áreas de interesse, o que lhes causa certo descrédito no potencial da disciplina e a visão de que é apenas "mais uma matéria a vencer". Desconstruir essa visão depende da criação de estratégias docentes favoráveis, personalizadas e que instiguem maior protagonismo por parte do próprio estudante, como indicado no Quadro 1, desenvolvido a partir do Plano de Ensino da disciplina.

Quadro 1 - Proposta e objetivos de aprendizagem da avaliação

\begin{tabular}{|l|ll|}
\hline Objetivos & $\begin{array}{l}\text { Propostas para alcançar a } \\
\text { aprendizagem }\end{array}$ & ara \\
\hline
\end{tabular}




\begin{tabular}{|c|c|}
\hline $\begin{array}{l}\text { Proporcionar o engajamento dos conteúdos } \\
\text { trabalhados em sala de aula com problemas } \\
\text { aplicados; }\end{array}$ & $\begin{array}{l}\text { Trabalhos em grupos; } \\
\begin{array}{l}\text { Orientações extraclasse guiadas por } \\
\text { rubricas; }\end{array}\end{array}$ \\
\hline $\begin{array}{l}\text { Possibilitar aos alunos uma visão crítica da } \\
\text { teoria e prática, com sua vida profissional; }\end{array}$ & $\begin{array}{l}\text { Contato dos alunos com docentes de } \\
\text { outras disciplinas em busca de }\end{array}$ \\
\hline $\begin{array}{l}\text { Desenvolver um ambiente de debates e } \\
\text { disseminação de aplicações durante a } \\
\text { apresentação dos seminários; }\end{array}$ & $\begin{array}{l}\text { aplicações e ideias; } \\
\text { Busca de aplicações em diferer } \\
\text { contextos; }\end{array}$ \\
\hline $\begin{array}{l}\text { companhar o pr } \\
\text { os alunos durant }\end{array}$ & $\begin{array}{l}\text { Desenvolvimento de um código } \\
\text { linguagem de programação para obter } \\
\text { resultados. }\end{array}$ \\
\hline
\end{tabular}

Fonte: Autores, 2020.

A implementação das rubricas se deu em quatro "fases", como representado na Figura 1 , tendo como objetivo acompanhar o processo de aprendizagem e engajamento dos alunos ao longo das etapas previstas. No que se refere à rubrica 1, os alunos, em horário extraclasse, tinham um encontro com o professor. Os encontros eram em grupos, com perguntas individuais efetuadas pelo professor, nos quais os alunos apresentavam um tema/problema que gostariam de desenvolver. Essa escolha implicava em uma aplicação qualquer, envolvendo um ou mais métodos numéricos estudados em sala de aula. Já a rubrica 2 era voltada ao desenvolvimento do trabalho em si e, também, ocorria atividade extraclasse de mentoria com o professor. Nessa etapa, o trabalho deveria estar na fase final de elaboração, necessitando apenas alguns ajustes, seja no código de programação, seja no documento escrito.

A rubrica 3 tem o objetivo de nortear os alunos sobre as dimensões avaliadas durante a apresentação oral do seminário e a rubrica 4 é utilizada para a correção do trabalho escrito de socialização dos resultados, seguindo as recomendações detalhadas em um template disponibilizado para modelar as tarefas previstas. Além da relação presencial estabelecida em sala de aula e em horários de mentoria extraclasse disponibilizados pelo docente, a comunicação para o planejamento de desenvolvimento do seminário proposto acontece via orientações desenhadas e disponibilizadas no sistema de gestão da aprendizagem Moodle.

Foram consideradas na elaboração das rubricas seis níveis: do excelente, com nota (10) ao insuficiente, com nota (0). Os pesos (pontuações) atribuídos às rubricas foram iguais para todas, de forma a não haver hierarquização de nenhuma fase sobre as demais, como forma de 
evidenciar aos alunos que todas as etapas eram igualmente importantes. A Figura 1 representa as proposições das rubricas em cada estágio já mencionado.

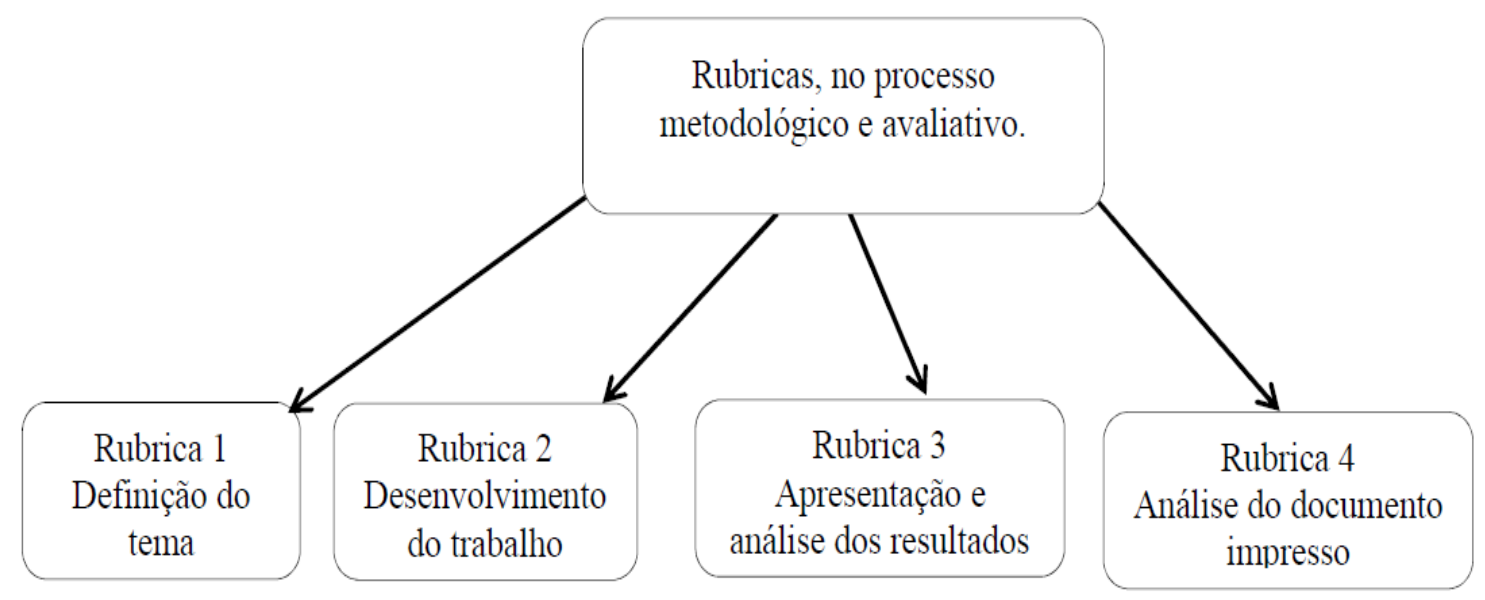

Fonte: Autores, 2020.

Figura 1 - Tipos de rubricas em cada estágio avaliativo

A pesquisa desenvolvida neste trabalho tem o foco na intervenção pedagógica (DAMIANI et al., 2013) e se vale tanto do viés quantitativo quanto do qualitativo, como predominância do primeiro sobre o segundo. Os dados quantitativos permitiram mapear os desempenhos discentes de forma comparativa entre os semestres em que as rubricas ainda não haviam sido implementadas $(2016.2,2017.1,2017.2)$ e os semestres em que as rubricas foram desenvolvidas e utilizadas (2018.2, 2019.1 e 2019.2), partindo das experiências prévias do docente no campo avaliativo, do embasamento teoricamente orientado e da pesquisa colaborativa, pautando o caráter fundamentalmente responsivo da pesquisa a partir da prática docente.

Nesse sentido, o olhar qualitativo é complementar ao quantitativo porque sendo uma abordagem intervencionista, os aspectos contextuais e a imersão dos pesquisadores no cenário da pesquisa permitem ampliar a compreensão da experiência avaliativa empreendida e remodelá-la a cada nova intervenção. A Figura 2 representa o contexto geral do número de alunos, semestres e cursos envolvidos. Ressalta-se que, sendo Cálculo Numérico uma disciplina de caráter multicurso (BLASS; IRALA, 2020a, 2020b), ou seja, as vagas nas turmas são ocupadas por quaisquer discentes que as solicitem no ato da matrícula, não havendo coincidência na representatividade de cursos em cada um dos semestres em que ela é 
ofertada, o que justifica a ausência de participantes de alguns cursos em alguns dos semestres em análise.

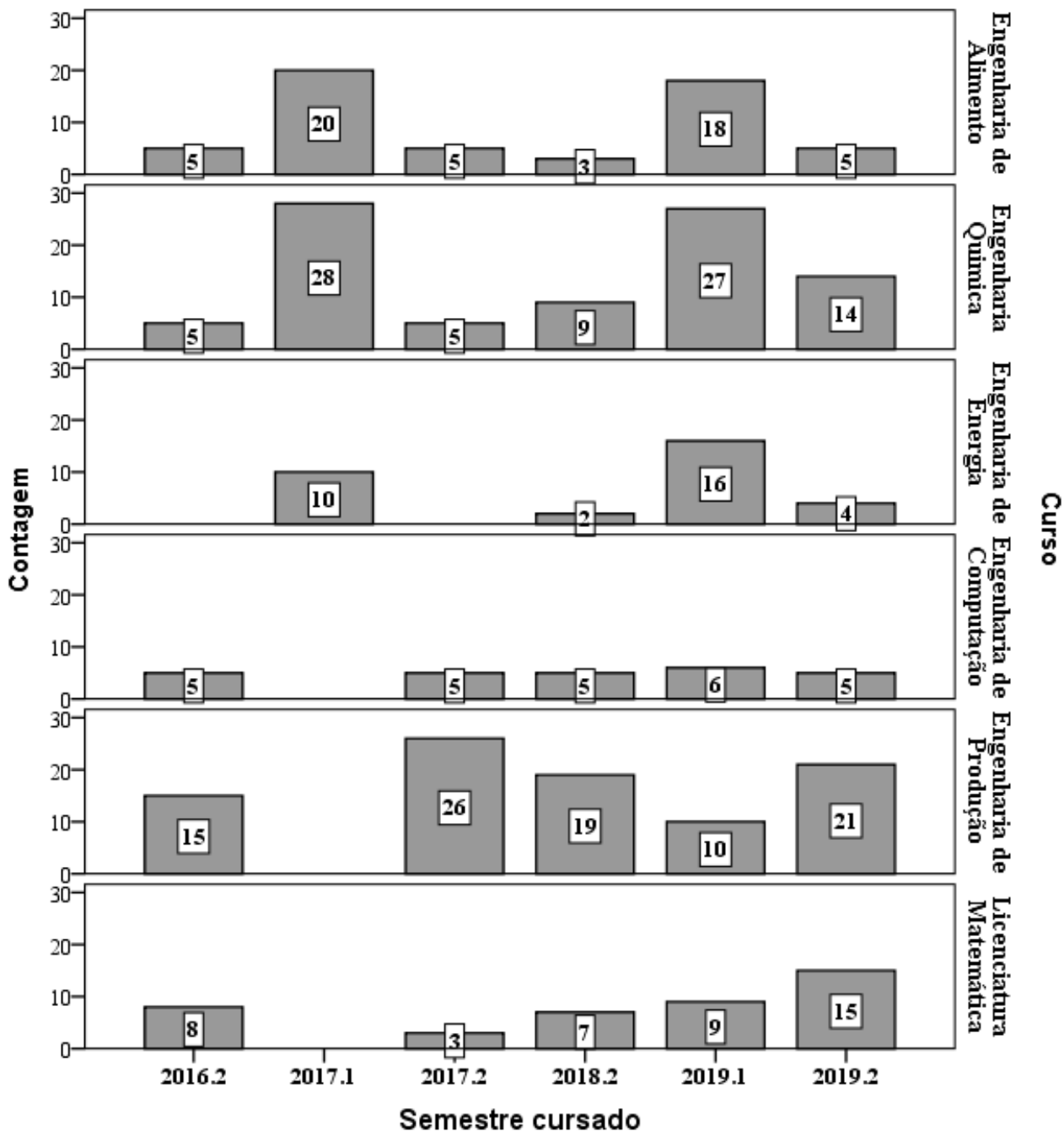

Fonte: Autores, 2020.

Figura 2 - Público-alvo da pesquisa

Os dados analisados advêm: a) dos desempenhos dos alunos nos três semestres em que não foram avaliados com as rubricas, havendo dois eixos avaliativos focados nos resultados, a apresentação oral e o trabalho impresso; b) dos desempenhos dos alunos nos três semestres em que a rubrica foi adotada, compondo a nota em quatro eixos avaliativos: a) definição do tema; b) desenvolvimento do trabalho; c) apresentação oral; d) documento final impresso. 
As análises de dados das amostras totalizaram 305 estudantes. O software utilizado foi o SPSS $^{\circledR}$ versão 20. Foram realizadas análises envolvendo estatística descritiva, testes de normalidade Shapiro-Wilk e Levene (teste F) para verificar a homogeneidade das variâncias das amostras, teste não-paramétrico de Kruskal-Wallis para verificar se há ou não diferenças no uso das rubricas e análise post hoc de Bonferroni, que examinou as diferenças entre os semestres (FIELD, 2009). Os resultados são apresentados para cada rubrica e semestre na seção seguinte, enfatizando o desempenho e, de forma complementar, observações realizadas pelo docente e percepções dos estudantes, quando pertinente.

\section{RESULTADOS E DISCUSSÕES}

O Quadro 2 representa a rubrica 1, utilizada pelo docente durante a mentoria voltada à determinação do tema.

Quadro 2 - Rubrica para definição do tema

\begin{tabular}{|l|l|}
\hline Dimensões & Escolha do Problema \\
\hline $\begin{array}{l}\text { Excelente } \\
\text { (10) }\end{array}$ & $\begin{array}{l}\text { Justifica a escolha do problema, levanta hipóteses que serão trabalhadas (e } \\
\text { métodos utilizados) e evidencia articulação para discutir o problema proposto } \\
\text { pelo grupo (ou individual), demonstra ter feito várias leituras em fontes } \\
\text { confiáveis (artigos e livros científicos, etc. Obs: citar as leituras que foram } \\
\text { realizadas), respondendo com propriedade os questionamentos feitos pelo } \\
\text { docente durante a reunião. }\end{array}$ \\
\hline $\begin{array}{l}\text { Muito bom } \\
(\mathbf{9})\end{array}$ & $\begin{array}{l}\text { Justifica a escolha do problema, levanta hipóteses que serão trabalhadas (e } \\
\text { métodos utilizados) e evidencia articulação para discutir o problema proposto } \\
\text { pelo grupo (ou individual), demonstra ter feito leituras em fontes confiáveis } \\
\text { (artigos e livros científicos, etc. Obs: citar as leituras que foram realizadas), } \\
\text { mas ainda não tem total clareza sobre o problema e pode titubear perante os } \\
\text { questionamentos feitos pelo docente durante a reunião. }\end{array}$ \\
\hline Bom (8) & $\begin{array}{l}\text { Justifica a escolha, levanta hipóteses e evidencia articulação para discutir o } \\
\text { problema proposto pelo grupo (ou individual), tem alguma ideia em relação } \\
\text { aos métodos, realizou poucas leituras - no mínimo 3 (artigos, livros } \\
\text { científicos, etc. Obs: citar as leituras que foram realizadas) sobre o assunto, } \\
\text { não consegue responder com clareza os questionamentos feitos pelo docente } \\
\text { durante a reunião. }\end{array}$ \\
\hline $\begin{array}{l}\text { Aceitável } \\
(6-7)\end{array}$ & $\begin{array}{l}\text { Justifica a escolha, levanta hipóteses e evidencia alguma articulação para } \\
\text { discutir o problema proposto pelo grupo (ou individual), realizou poucas } \\
\text { leituras - no máximo } 3 \text { (artigos, livros científicos, etc. Obs: citar as leituras } \\
\text { que foram realizadas), mas não consegue responder com clareza os } \\
\text { questionamentos feitos pelo docente durante a reunião e ainda não sabe quais } \\
\text { métodos pretende adotar. }\end{array}$ \\
\hline $\begin{array}{l}\text { Inadequado } \\
(\mathbf{0 - 5 )}\end{array}$ & $\begin{array}{l}\text { Ainda está se apropriando da proposta, mais escutando os colegas e o } \\
\text { professor do que se posicionamento sobre o problema proposto. Espera que o } \\
\text { professor e/ou os colegas lhe deem soluções. }\end{array}$ \\
$\begin{array}{l}\text { Não apresentou qualquer ideia ou não compareceu. } \\
\text { fonte }\end{array}$ \\
\hline
\end{tabular}

Fonte: Autores, 2020. 
A Tabela 1 representa os dados obtidos para os três semestres em que a rubrica 1 foi implementada.

Tabela 1 - Desempenhos na definição do tema (rubrica 1)

\begin{tabular}{|c|c|c|c|c|c|c|}
\hline Semestre & $\mathbf{N}^{\mathbf{0}}$ alunos & Média & Mediana & Desvio padrão & Mínimo & Máximo \\
\hline $\mathbf{2 0 1 8 . 2}$ & 42 & 9,9 & 10,0 & 0,3 & 9,0 & 10,0 \\
\hline $\mathbf{2 0 1 9 . 1}$ & 75 & 9,8 & 10,0 & 0,7 & 5,0 & 10,0 \\
\hline $\mathbf{2 0 1 9 . 2}$ & 53 & 9,8 & 10,0 & 0,5 & 8,0 & 10,0 \\
\hline
\end{tabular}

Fonte: Autores, 2020.

Através da Tabela 1, podemos afirmar, pelos valores de média e de mediana que a definição do tema não gerou muitas dificuldades para os alunos. O valor mínimo cinco para o semestre 2091.1 proporcionou uma variação maior no desvio padrão. Nessa etapa, de forma geral, os alunos conseguiram apresentar ideias claras, buscaram diversas fontes e até mesmo recorreram a outros professores de diferentes áreas, até chegar a um consenso da definição do tema que melhor se ajustasse à proposta.

O Quadro 3 representa a rubrica 2, adotada durante o segundo encontro de mentoria, em que os alunos apresentavam ao docente o desenvolvimento do trabalho. Nesse encontro, o trabalho deveria estar em tese finalizado, apenas para o docente sugerir mudanças e aprimoramentos para as etapas seguintes.

Quadro 3 - Rubrica 2, adotada para o desenvolvimento do trabalho

\begin{tabular}{|l|l|}
\hline \multicolumn{1}{|c|}{ Dimensões } & \multicolumn{1}{|c|}{ Desenvolvimento do trabalho } \\
\hline Excelente & $\begin{array}{l}\text { Fala com bastante propriedade as ideias desenvolvidas para a resolução do } \\
\text { problema, tem clareza em relação aos métodos e conceitos adotados (sabe } \\
\text { explicar quando questionado). Sabe apresentar o passo-a-passo e as decisões } \\
\text { tomadas até então pelo grupo (ou individual), em qualquer âmbito do } \\
\text { desenvolvimento do trabalho. O trabalho se encontra no template } \\
\text { disponibilizado, o código deve gerar os resultados sem erros. O trabalho deve } \\
\text { vir na versão final (esse item será para que o professor possa dar sugestões). }\end{array}$ \\
\hline Muito bom & $\begin{array}{l}\text { Consegue apresentar as ideias desenvolvidas para a resolução do problema, } \\
\text { entende os métodos e conceitos adotados (sabe explicar quando questionado). } \\
\text { Sabe apresentar o passo-a-passo e as decisões tomadas até então pelo grupo (ou } \\
\text { individual), em qualquer âmbito do desenvolvimento do trabalho. O trabalho se } \\
\text { encontra no template disponibilizado, o código deve estar gerando os resultados. } \\
\text { O trabalho deve vir na versão final (esse item será para que o professor possa } \\
\text { dar sugestões). }\end{array}$ \\
\hline Bom (8) & $\begin{array}{l}\text { Consegue apresentar as ideias desenvolvidas para a resolução do problema, } \\
\text { demonstra entender os métodos e conceitos adotados (sabe explicar em alguma } \\
\text { medida quando questionado). Sabe apresentar o passo-a-passo (em partes) e a } \\
\text { maioria das decisões tomadas até então pelo grupo (ou individual). O trabalho se } \\
\text { encontra no template disponibilizado, o código deve estar gerando os resultados. } \\
\text { O trabalho deve vir na versão final (esse item será para que o professor possa } \\
\text { dar sugestões). }\end{array}$ \\
\hline
\end{tabular}




\begin{tabular}{|l|l|}
\hline $\begin{array}{l}\text { Aceitável } \\
(6-7)\end{array}$ & $\begin{array}{l}\text { Fala sobre as ideias desenvolvidas para a resolução do problema, consegue } \\
\text { demonstrar algum entendimento sobre os métodos e conceitos adotados (sabe } \\
\text { explicar em alguma medida quando questionado). Sabe apresentar o passo-a- } \\
\text { passo (em partes) e as decisões tomadas até então pelo grupo (ou individual). O } \\
\text { trabalho se encontra no template disponibilizado e o código deve estar gerando } \\
\text { alguns resultados, ainda necessitando ajustes. O trabalho deve vir na versão } \\
\text { final (esse item será para que o professor possa dar sugestões). }\end{array}$ \\
\hline $\begin{array}{l}\text { Inadequado } \\
\text { (0- 5) }\end{array}$ & $\begin{array}{l}\text { Fala sem propriedade sobre a resolução do problema, tem pouca clareza em } \\
\text { relação aos métodos e conceitos adotados. Não consegue apresentar o passo-a- } \\
\text { passo e as decisões tomadas até então pelo grupo (ou individual), nos diferentes } \\
\text { âmbitos do desenvolvimento do trabalho. O trabalho se encontra parcialmente no } \\
\text { template e pode também haver problemas no código. O trabalho deve vir na } \\
\text { versão final (esse item será para que o professor possa dar sugestões). }\end{array}$ \\
\hline Insuficiente & $\begin{array}{l}\text { Não desenvolveu nada ou quase nada do trabalho ou não compareceu. } \\
\text { (0) }\end{array}$ \\
\hline
\end{tabular}

Fonte: Autores, 2020.

A Tabela 2 representa a descrição estatística dos resultados para a rubrica 2, nos semestres em que foi adotada.

Tabela 2 - Desempenhos para o desenvolvimento do trabalho (rubrica 2)

\begin{tabular}{|c|c|c|c|c|c|c|}
\hline Semestre & $\mathbf{N}^{\mathbf{0}}$ alunos & Média & Mediana & Desvio padrão & Mínimo & Máximo \\
\hline $\mathbf{2 0 1 8 . 2}$ & 42 & 9,9 & 10,0 & 0,2 & 9,0 & 10,0 \\
\hline $\mathbf{2 0 1 9 . 1}$ & 75 & 8,4 & 8,0 & 1,0 & 6,5 & 10,0 \\
\hline $\mathbf{2 0 1 9 . 2}$ & 53 & 9,3 & 10,0 & 0,9 & 7,0 & 10,0 \\
\hline
\end{tabular}

Fonte: Autores, 2020.

Foi possível identificar, através de média, mediana e desvio padrão, que existiu mais dificuldades no desenvolvimento do trabalho, já que essa etapa envolve competências mais complexas do que a etapa anterior, como a primeira versão do documento escrito e o desenvolvimento do código. A Tabela 2 descreve que os alunos do semestre 2019.1 tiverem maiores dificuldades. Uma percepção que aparece em todos os encontros diz respeito às dificuldades recorrentes na elaboração do código (que tem a função de gerar os resultados para os trabalhos dos seminários) em alguma linguagem de programação a escolha dos estudantes (há, em geral, predomínio das linguagens $\mathrm{C}, \mathrm{C}++$ e do software SCILAB). A Figura 3 representa, apenas de forma ilustrativa, os resultados de uma das respostas de um questionário coletado junto a turma 2019.2 diante da pergunta: "Considero que, para a elaboração do seminário, a implementação do código foi o que considerei o mais difícil de realizar". Ressalta-se que o questionário original também apresentava outras perguntas semelhantes, relacionadas às demais fases da proposta avaliativa. Ilustra-se aqui apenas a 
reposta vinculada ao aspecto considerado mais problemático de todo o processo. A discussão detalhada desses dados coletados em questionários pode ser encontrada em trabalhos já publicados (BLASS; IRALA 2020a; BLASS; GOERGEN; IRALA, 2020).
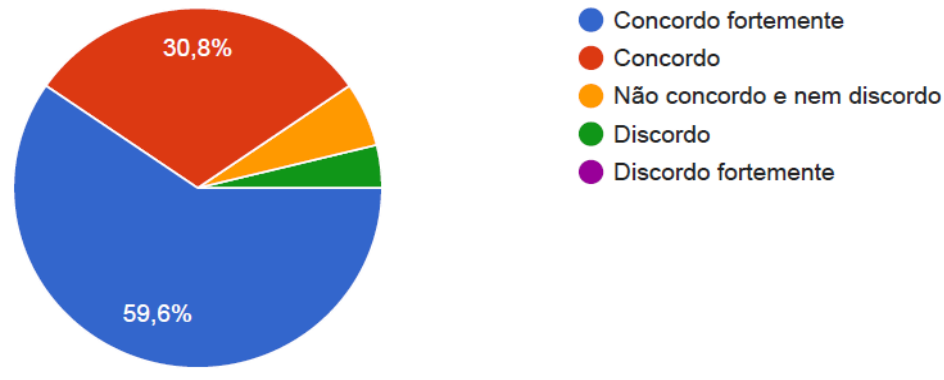

Fonte: Autores, 2020.

Figura 3 - Dados referentes ao desenvolvimento do código

De acordo com a Figura 3, 90,4\% concordava ou concordava fortemente que, de todas as etapas, o desenvolvimento do código oferece maior dificuldade que as demais tarefas abrangentes para a realização do trabalho. A disciplina dispõe de aulas de programação complementares para auxiliar na execução dos resultados; porém, há indícios, depois de alguns semestres com recorrentes relatos de dificuldades nesse aspecto, de que o conhecimento prévio dos estudantes (os quais, em sua maioria, apenas cursaram uma disciplina de Algoritmos e Programação) podem não ser suficientes para executar com segurança os procedimentos que requerem alguma experiência em linguagens de programação. As percepções do docente sobre esse aspecto e a observação qualitativa das dificuldades mapeadas junto aos discentes podem contribuir para aperfeiçoar o delineamento curricular, de forma a suprir essa lacuna identificada na prática. O Quadro 4 representa as dimensões observadas pela rubrica 3, que enfatiza a apresentação oral dos resultados.

Quadro 4: Rubrica 3 adotada para a avaliação da apresentação oral

\begin{tabular}{|l|l|}
\hline Dimensões & APRESENTAÇÃO E ANÁLISE DOS RESULTADOS \\
\hline Excelente & $\begin{array}{l}\text { O conteúdo da apresentação dialoga plenamente com o trabalho escrito. O aluno } \\
\text { explica de maneira clara, não se prende à leitura, demonstra conhecimento } \\
\text { consistente do que foi produzido pelo grupo (ou individual). Suas respostas às } \\
\text { eventuais perguntas são coerentes com o trabalho e evidenciam compreensão } \\
\text { aprofundada do problema em todos os níveis requeridos. Fez uso adequado do } \\
\text { tempo destinado. Resultados completos, organizados de forma excelente e } \\
\text { aprofundados, com as devidas análises de resultados e métodos, presentes de forma } \\
\text { clara nos recursos escolhidos (gráficos, tabelas, quadros, etc. - todos numerados). } \\
\text { Consegue fazer plena conexão do conteúdo trabalhado em sala de aula com a } \\
\text { aplicação. }\end{array}$ \\
\hline
\end{tabular}




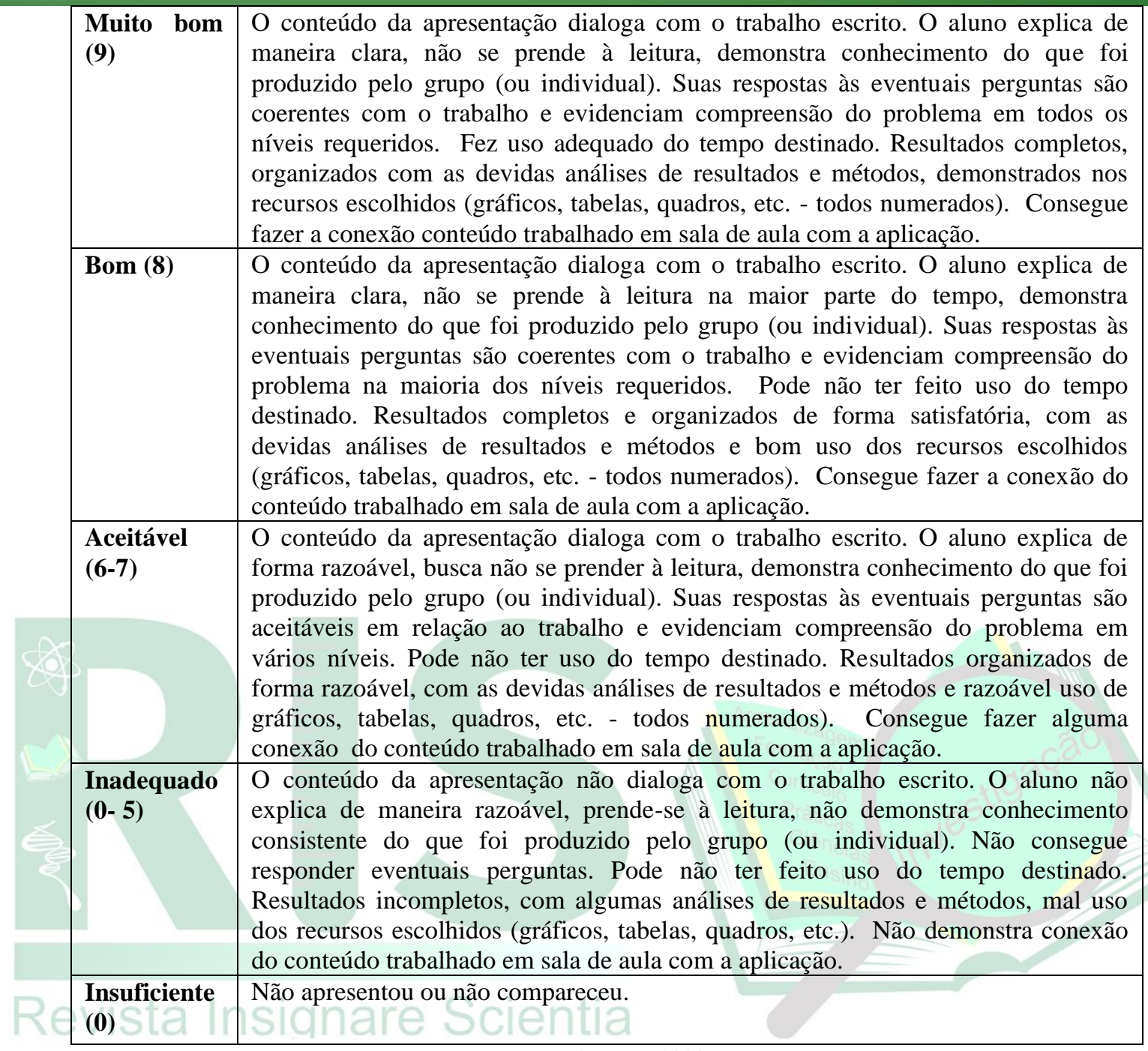

Fonte: Autores, 2020.

A Tabela 3 representa os resultados referentes à apresentação oral dos seminários. Destaca-se que para os semestres 2016.2, 2017.1 e 2017.2 não foi utilizada a rubrica, apenas um checklist (no checklist continha algumas observações que o professor achava pertinentes para uma apresentação). O checklist era apenas usado pelo professor e os alunos não tinham acesso a ele. Observações realizadas em notas de campo pelo professor apontam em relação a esses semestres para uma maior ansiedade e insegurança nas apresentações por parte de alguns estudantes, menor domínio sobre o assunto e resultados (gráficos, tabelas e quadros) menos organizados.

Tabela 3 - Desempenhos em apresentação dos seminários

\begin{tabular}{|c|c|c|c|c|c|c|}
\hline Semestre & N $^{0}$ alunos & Média & Mediana & Desvio padrão & Mínimo & Máximo \\
\hline 2016.2-SR & 38 & 6,9 & 7,0 & 1,3 & 4,0 & 9,0 \\
\hline 2017.1-SR & 56 & 8,0 & 8,5 & 1,1 & 5,0 & 9,5 \\
\hline
\end{tabular}




\begin{tabular}{|l|c|c|c|c|c|c|}
\hline 2017.2-SR & 41 & 7,9 & 9,0 & 1,5 & 5,0 & 9,6 \\
\hline 2018.2-CR & 42 & 8,8 & 9,0 & 1,2 & 6,0 & 10,0 \\
\hline 2019.1-CR & 75 & 8,2 & 8,0 & 0,7 & 6,0 & 9,0 \\
\hline 2019.2-CR & 53 & 9,3 & 9,0 & 0,7 & 8,0 & 10,0 \\
\hline
\end{tabular}

Fonte: Autores, 2020.

Pela Tabela 3, pode-se observar que as médias relativas à apresentação oral se diferem entre os semestres em que foram utilizadas as rubricas em comparação com os semestres sem rubricas. Os valores mínimos também se diferem, assim com os máximos. Com $95 \%$ de significância, foi testada a normalidade dos dados, através do teste de Shapiro-Wilk. O teste se destaca pela sua eficiência e flexibilidade ao se tratar de diferentes tamanhos de amostra (SHAPIRO; WILK, 1965; TORMAN; COSTA; RIBOLDI, 2012). O teste de Levene foi usado para verificar se há homogeneidade da variância (FIELD, 2009). Os resultados de Shapiro-Wilk não representam uma distribuição normal, ou seja, para 2016.2 (D (38) = 0,936; Valor-p < 0,05), 2017.1 (D (56) = 0,853; Valor-p < 0,001), $2017.2(\mathrm{D}(41)=0,848$; Valor-p < 0,001), $2018.2(\mathrm{D}(42)=0,793$; Valor-p < 0,001), 2019.1(D (75) = 0,826; Valor-p < 0,001) e $2019.2(\mathrm{D}(53)=0,770 ;$ Valor-p $<0,001)$.

O resultado do teste de Levene, baseado nas médias dos semestres (FIELD, 2009, p. $119)$, $(\mathrm{F}(5,299)=10,230$; Valor-p < 0,05), mostrou que a distribuição dos dados não possui normalidade e não há homogeneidade das variâncias. A partir dos resultados de Shapiro-Wilk e Levene, foi realizado o teste não-paramétrico de Kruskal-Wallis (FIELD, 2009) com a seguintes hipóteses: H0: não há diferença nas notas dos semestres em que foram usadas as rubricas comparadas com os semestres em que não foram usadas; H1: Há diferença nas notas dos semestres em que foram usadas as rubricas em comparação com os semestres em que não foram usadas. O teste de Kruskal-Wallis mostrou que há efeito das notas dos semestres com o uso das rubricas $(\mathrm{H}(5)=98,263$; Valor-p <0,001). A partir do resultado de KruskalWallis, foi realizada uma análise post hoc, cuja finalidade é a comparação em pares planejados, a partir de diferentes combinações (FIELD, 2009).

O SPSS ${ }^{\circledR}$ faz o post hoc usando comparações em pares (nesse caso, com todos os semestres), partindo da abordagem de Dunn-Bonferroni (FIELD, 2009). O post hoc mostrou que o desempenho no semestre 2016.2 é diferente dos semestres em que foram usadas as rubricas; o semestre de 2017.1 é diferente dos semestres 2018.2 e 2019.2, mas não difere do semestre 2019.1; o semestre 2017.2 foi diferente do semestre 2019.2 e 2018.2, mas não houve 
Quadro 5 - Rubrica 4, para a avaliação do trabalho impresso

\begin{tabular}{|c|c|}
\hline Dimensões & ANÁLISE DO DOCUMENTO IMPRESSO \\
\hline $\begin{array}{l}\text { Excelente } \\
\text { (10) }\end{array}$ & $\begin{array}{l}\text { No documento impresso são inseridas as referências de forma plenamente adequada. } \\
\text { Contempla com clareza os objetivos e a justificativa. O texto é escrito de maneira } \\
\text { clara, contextualização adequada e há demonstração de conhecimento consistente do } \\
\text { que foi produzido pelo grupo (ou individual). Segue o template disponibilizado. } \\
\text { Resultados completos, organizados de forma excelente e aprofundados, com precisão } \\
\text { de detalhes e coerentes com os métodos utilizados, fontes e recursos gráficos, tabelas, } \\
\text { quadros, etc. (todos numerados). Esses recursos são bem analisados quando aparecem } \\
\text { no texto. Apresentação da opinião sobre o trabalho realizado (na conclusão) de } \\
\text { forma consistente e crítica. }\end{array}$ \\
\hline $\begin{array}{l}\text { Muito bom } \\
\text { (9) }\end{array}$ & $\begin{array}{l}\text { No documento impresso são inseridas as referências, na maioria das vezes, de forma } \\
\text { plenamente adequada. Contempla os objetivos e justificativa. O texto é produzido de } \\
\text { maneira clara, contextualizada e demonstração de conhecimento consistente do que } \\
\text { foi produzido pelo grupo (ou individual). Segue o template disponibilizado. } \\
\text { Resultados completos, bem organizados, detalhes e coerentes dos métodos utilizados, } \\
\text { fontes e recursos - gráficos, tabelas, quadros, etc. (todos numerados). Esses recursos } \\
\text { são bem analisados quando aparecem no texto. Apresentação da opinião sobre o } \\
\text { trabalho realizado (na conclusão). }\end{array}$ \\
\hline Bom (8) & $\begin{array}{l}\text { No documento impresso são inseridas as referências, na maioria das vezes, de forma } \\
\text { adequada. Contempla os objetivos e justificativa. O texto é escrito de forma clara, há } \\
\text { contextualização e demonstração do conhecimento do que foi produzido pelo grupo } \\
\text { (ou individual). Segue em partes o template disponibilizado. Resultados completos, } \\
\text { organizados de forma satisfatória, coerentes com os métodos utilizados, fontes e } \\
\text { recursos gráficos, tabelas, quadros, etc. (todos numerados). Esses recursos são bem } \\
\text { analisados quando aparecem no texto. Apresentação da opinião sobre o trabalho } \\
\text { realizado (na conclusão). }\end{array}$ \\
\hline $\begin{array}{l}\text { Aceitável } \\
(6-7)\end{array}$ & $\begin{array}{l}\text { No documento impresso são inseridas as referências, porém pode haver alguns } \\
\text { problemas nesse aspecto. Contempla os objetivos e justificativa. O texto } \\
\text { contextualiza de forma razoável e evidencia algum conhecimento do que foi } \\
\text { produzido pelo grupo (ou individual). Segue em partes o template disponibilizado. } \\
\text { Resultados pouco aprofundados (explicados), mas coerentes com os métodos } \\
\text { utilizados, fontes e recursos gráficos, tabelas, quadros, etc. (todos numerados). Esses } \\
\text { recursos são relativamente bem organizados no texto. Apresentação da opinião de } \\
\text { forma razoável sobre o trabalho realizado (na conclusão). }\end{array}$ \\
\hline $\begin{array}{l}\text { Inadequado } \\
(0-5)\end{array}$ & $\begin{array}{l}\text { Os resultados não condizem com os objetivo(s) e justificativa. Não traz resultados ou } \\
\text { estão incompletos e insuficientes. }\end{array}$ \\
\hline $\begin{array}{l}\text { Insuficiente } \\
\text { (0) }\end{array}$ & 10. \\
\hline
\end{tabular}

Fonte: Autores, 2020.

A Tabela 4 representa as análises referentes aos semestres em que foram usadas as rubricas versus os semestres em que foi utilizado apenas um checklist elaborado pelo docente.

Tabela 4 - Desempenhos no trabalho impresso

\begin{tabular}{|c|c|c|c|c|c|c|}
\hline Semestre & $\mathbf{N}^{\mathbf{0}}$ alunos & Média & Mediana & Desvio padrão & Mínimo & Máximo \\
\hline 2016.2-SR & 38 & 7,6 & 8,0 & 1,1 & 4,0 & 8,5 \\
\hline 2017.1-SR & 56 & 7,8 & 8,0 & 1,2 & 5,0 & 9,5 \\
\hline
\end{tabular}




\begin{tabular}{|l|l|l|l|l|l|l|}
\hline 2017.2-SR & 41 & 7,7 & 8,5 & 1,7 & 5,0 & 10,0 \\
\hline 2018.2-CR & 42 & 8,7 & 9,0 & 1,3 & 6,0 & 10,0 \\
\hline 2019.1-CR & 75 & 8,3 & 8,0 & 0,8 & 7,0 & 10,0 \\
\hline 2019.2-CR & 53 & 8,6 & 9,0 & 1,3 & 6,0 & 10,0 \\
\hline
\end{tabular}

A Tabela 4 mostrou que os semestres em que não foram usadas as rubricas apresentaram médias, medianas, mínimos e máximos inferiores aos semestres em que as rubricas foram usadas para a avaliação do trabalho impresso, enfatizando a importância das rubricas como estratégia docente.

Os resultados de Shapiro-Wilk não representaram uma distribuição normal, ou seja, para 2016.2 (D (38) = 0,602; Valor-p < 0,001), 2017.1 (D (56) =0,925; Valor-p < 0,05), 2017.2 (D (41) =0,896; Valor-p < 0,05), 2018.2 (D (42) =0,819; Valor-p < 0,001), 2019.1(D (75) =0,917; Valor-p < 0,001) e 2019.2 (D (53) =0,831; Valor-p < 0,001), assim como o teste de Levene $(F(5,299)=16,243$; Valor-p < 0,05). Os testes mostraram que a distribuição dos dados não possui normalidade e não há homogeneidade das variâncias, o que leva para a realização de testes não-paramétricos.

Foi realizado o teste não-paramétrico de Kruskal-Wallis para verificar as diferenças entre os semestres com e sem o uso de rubricas para avaliar o trabalho impresso. O teste mostrou que há efeito dos semestres com o uso das rubricas $(\mathrm{H}(5)=34,460$; Valor-p <0,001). $\mathrm{O}$ post hoc mostrou que para o semestre de 2016.2 o desempenho é diferente dos semestres em que foram usadas as rubricas 2018.2 e 2019.2, mas não para 2019.1; para o semestre 2017.1 foi diferente para os semestres 2018.2 e 2019.2 e não para 2019.1. Por fim, para o semestre 2017.2 foi diferente para o semestre 2018.2 e não para 2019.1 e 2019.2.

A Tabela 5 representa as médias das notas dos seminários avaliados com as rubricas (nesse caso, as médias das quatro notas referentes às quatro rubricas), comparadas às médias das notas dos seminários sem o uso das rubricas (nesse caso, a média das duas notas, referentes à apresentação e ao trabalho impresso).

Tabela 5 - Notas finais dos seminários

\begin{tabular}{|c|c|c|c|c|c|c|}
\hline Semestre & $\mathbf{N}^{\mathbf{0}}$ alunos & Média & Mediana & Desvio padrão & Mínimo & Máximo \\
\hline $\mathbf{2 0 1 6 . 2}$ & 38 & 7,3 & 7,6 & 1,2 & 4,0 & 8,8 \\
\hline $\mathbf{2 0 1 7 . 1}$ & 56 & 7,9 & 8,3 & 1,1 & 5,0 & 9,3 \\
\hline
\end{tabular}




\begin{tabular}{|c|c|c|c|c|c|c|}
\hline $\mathbf{2 0 1 7 . 2}$ & 41 & 7,8 & 8,0 & 1,5 & 5,0 & 9,8 \\
\hline $\mathbf{2 0 1 8 . 2}$ & 42 & 9,3 & 9,5 & 0,6 & 7,8 & 10,0 \\
\hline $\mathbf{2 0 1 9 . 1}$ & 75 & 8,7 & 8,9 & 0,5 & 7,5 & 9,5 \\
\hline $\mathbf{2 0 1 9 . 2}$ & 53 & 9,3 & 9,5 & 0,6 & 8,2 & 10,0 \\
\hline
\end{tabular}

Os resultados de Shapiro-Wilk não representaram uma distribuição normal, ou seja, para 2016.2 (D (38) =0,814; Valor-p < 0,001), 2017.1 (D (56) =0,897; Valor-p < 0,001), 2017.2 (D (41) =0,910; Valor-p < 0,05), 2018.2 (D (42) =0,876; Valor-p < 0,001), 2019.1(D (75) =0,924; Valor-p < 0,001) e 2019.2 (D (53) =0,870; Valor-p < 0,001), assim como o teste de Levene $(F(5,299)=10,024$; Valor-p < 0,05). Os testes mostraram que a distribuição dos dados não possui normalidade e não há homogeneidade das variâncias.

Foi realizado o teste não paramétrico de Kruskal-Wallis (FIELD, 2009) para verificar se há diferenças das médias das notas finais, com a soma de todas as etapas, nos semestres com e sem as rubricas. O teste mostrou que há efeito no desempenho dos semestres com o uso das rubricas $(\mathrm{H}(5)=128,906$; Valor-p <0,001). O post-hoc mostrou que, em média, o semestre 2016.2 é diferente dos semestres em que foram usadas as rubricas (2018.2, 2019.1 e 2019.2), bem como o semestre de 2017.1. O semestre 2017.2 é diferente de 2018.2 e 2019.2 e não do semestre 2019.1.

A Figura 4 representa a situação final de cada semestre, tendo como objetivo comparar os semestres que não tiveram avaliação com rubricas com os que adotaram a proposta. Entende-se que outras variáveis intervêm nesses resultados finais, além da adoção das rubricas; porém, o acompanhamento longitudinal e comparativo de cada experiência em que a disciplina é ofertada, por meio de registros reflexivos gravados em áudio produzidos pelo docente, questionários sistemáticos de avaliação das propostas didáticas adotadas ao final de cada semestre junto aos estudantes, além de conversas informais para coleta de feedback são elementos qualitativos importantes para indicar os benefícios da introdução das rubricas na prática avaliativa de Cálculo Numérico ao longo dos semestres avaliados. 


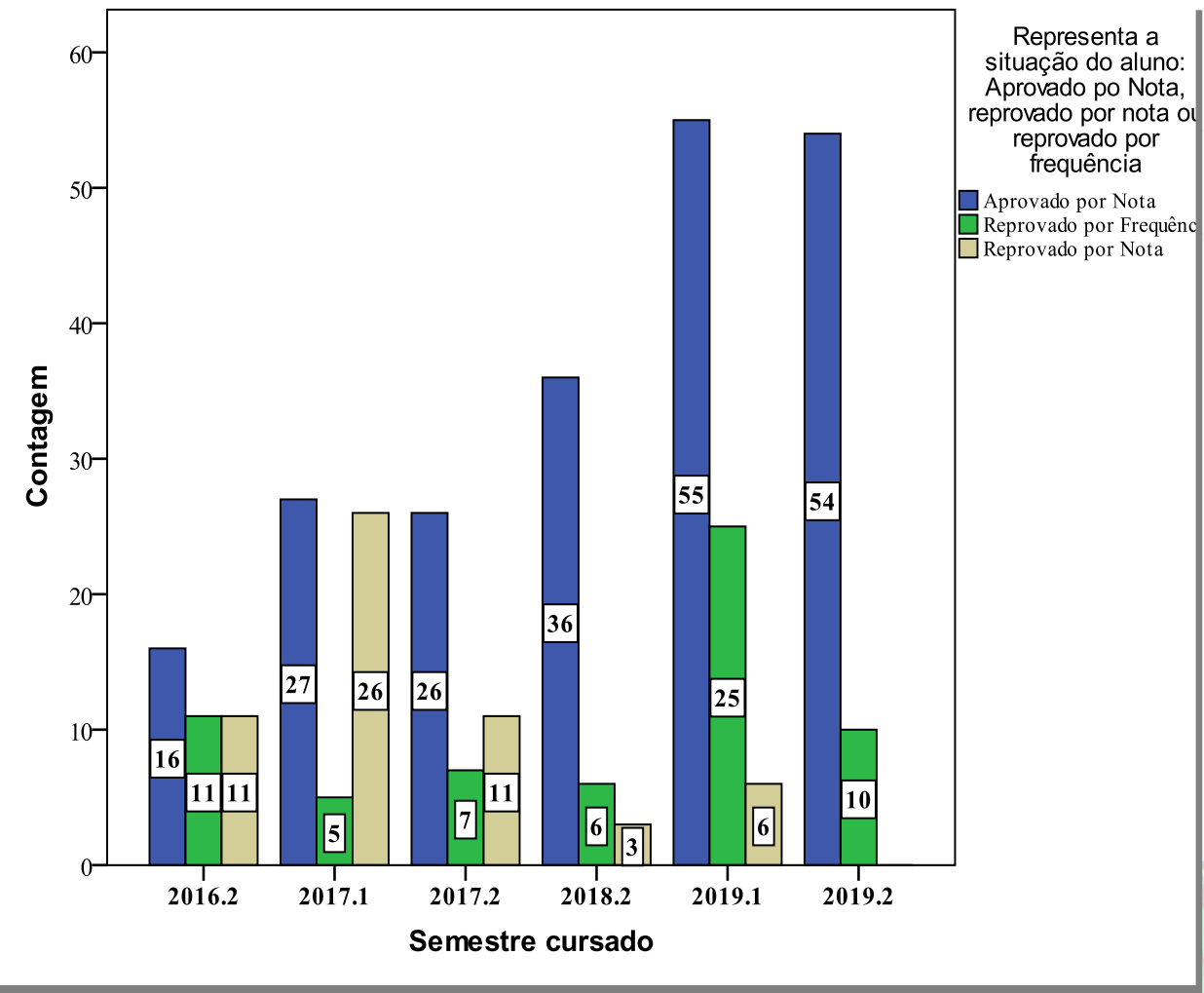

Fonte: Autores, 2020.

Figura 4 - Situação final dos alunos referentes a cada semestre

Através da Figura 4, é possível destacar que havia um maior índice de reprovação por nota, que aos poucos foi diminuindo significativamente com o uso das rubricas, mesmo que o conjunto das avaliações por rubricas represente apenas $30 \%$ do total das notas da disciplina. Identifica-se, com isso, que o uso das rubricas tem influência nesses novos índices, pelo fato de que o acompanhamento do processo realizado nas sessões de mentoria permite ampliar e qualificar a interação entre docente e discentes também nas demais atividades avaliativas propostas, pois o docente passa a mapear o perfil discente com um viés mais qualitativo do que é possível alcançar nas interações ocorridas apenas no contexto de sala de aula, como costumava acontecer anteriormente.

\section{CONSIDERAÇÕES FINAIS}

As rubricas tiveram um papel muito importante desde a sua implantação na disciplina de Cálculo Numérico. Foi possível estabelecer métricas para melhorar a qualidade dos trabalhos, explicação detalhada dos objetivos, critérios para avaliação discente, além de 
promover um sistema de avaliação mais coerente. Os alunos afirmaram que os feedbacks das rubricas, obtidos através das conversas informais realizadas com o docente, serviram como instrumento de reflexão e contribuíram para uma melhor comunicação entre estudantes e o professor.

A rubrica como estratégia avaliativa forneceu dados e informações que dão suporte à tomada de decisões futuras dos docentes que, a partir desse diagnóstico, podem repensar as metodologias empregadas em suas disciplinas para um acompanhamento da aprendizagem.

De acordo com a pesquisa, identificam-se algumas vantagens e desvantagens sobre o uso das rubricas: entre as desvantagens, o tempo dispendido para o seu desenvolvimento e também a dificuldade no estabelecimento dos critérios mais adequados e revisão constante do conteúdo das descrições; já como vantagens, a garantia ao docente de atribuir uma nota de modo mais preciso, justo e confiável, permitindo padronização, facilitação da autoavaliação, para que os alunos entendam de forma mais qualificada os seus desempenhos, sabendo, assim, em que aspectos devem focar seus esforços para geração de melhorias.

Em relação aos resultados obtidos a partir das análises efetuadas ao longo de seis semestres, constatou-se que o uso das rubricas deve ser mantido no componente de Cálculo Numérico. O post hoc mostrou que o uso das rubricas foi mais significativo, embora o semestre 2017.2 não tenha apresentado diferença em relação ao semestre 2019.1, o que pode ter ocorrido em razão da idiossincrasia do grupo. Para trabalhos futuros, tendo em vista que a intervenção através de rubricas se limitou a uma parcela do processo avaliativo, ou seja, foram usadas provas individuais para o restante das avaliações, pretende-se analisar a adoção de rubricas no conjunto de todas as tarefas propostas, ampliando a estratégia para todo o escopo da disciplina de Cálculo Numérico no contexto analisado. 
BLASS, Leandro; IRALA, Valesca Brasil. O uso da Aprendizagem Baseada em Problemas (PBL) como metodologia de ensino em aulas de Cálculo Numérico. Revista de Educação Matemática, v. 17, p. e020035, 2020a. DOI:

https://doi.org/10.37001/remat25269062v17id360.

BLASS, Leandro; IRALA, Valesca Brasil. Desenho avaliativo por rubricas em disciplina multicurso: análise de uma implementação piloto. HOLOS, v. 7, p. 1-24, 31 dez. 2020b. DOI: $10.15628 /$ holos.2020.9518.

BLASS, Leandro; GOERGEN, Guilherme; IRALA, Valesca Brasil. Análise das percepções e desempenhos discentes com a utilização do software $\mathbf{R}$ em uma disciplina de graduação de natureza multicurso. Revista Prática Docente, v. 5, n. 3, p. 1568-1592, 2020. DOI: https://doi.org/10.23926/RPD.2526-2149.2020.v5.n3.p1568-1592.id886.

BOSTON, Carol. Understanding Scoring Rubrics: A Guide for Teachers. Maryland: University of Maryland - ERIC Clearinghouse on Assessment and Evaluation, 1 ed., 2002.

BROOKHART, Susan. How to create and use rubrics for formative assessment and grading. Alexandria, Virginia (USA): ASCD, 2013.

CARLESS, David. Feedback loops and the longer-term: towards feedback spirals. Assessment \& Evaluation in Higher Education, v. 44, n. 5, p. 705-714, 2019. DOI: 10.1080/02602938.2018.1531108.

COBO, Alex Xavier Jerves. Elementos de Cálculo Numérico. Quito: Abya-Yala, 2008.

COOPER, Bruce; GARGAN, Ann. Rubrics in Education Old Term, New Meanings. Phi Delta Kappan; Bloomington, v. 91, n. 1, p. 54-55, set. 2009. Disponível em: http://facultycenter.ischool.syr.edu/wp-content/uploads/2012/02/Rubrics-in-Education1.pdf. Acesso em: 28 out. 2018.

DAMIANI, Magda et al. Discutindo pesquisas do tipo intervenção pedagógica. Cadernos de Educação (UFPel), v. 45, p. 57-67, 2013.

FIELD, Andy. Descobrindo a estatística usando o SPSS. Porto Alegre: Artmed, 2009.

GARCIA, Elena Cano. La evaluación por competencias en la educación superior. Revista de currículum y formación del profesorado, v.12, n.3, p. 1-16, 2008.

GATICA-LARA, Florina; URIBARREN-BERRUETA, Teresita del Niño Jesús. ¿Cómo elaborar una rúbrica?, Investigación en Educación Médica, v. 2, n. 1, p. 61-65, 2013. Disponível em: https://www.redalyc.org/articulo.oa?id=3497/349733230010. Acesso em: 19 fev. 2021. 
GORDILLO, Juan Jesús Torres; RODRÍGUEZ, Victor Hugo Perera. La rúbrica como instrumento pedagógico para la tutorización y evaluación de los aprendizajes en el foro online en educación superior. Pixel-Bit: Revista de medios y educación, n. 36, p. 141-149, 2010.

HOWELL, Rebecca. Grading rubrics: hoopla or help? Innovations in Education and Teaching International, v.51, n. 5, p. 400-410, 2014.

JÁCOME, Édgar Picón. La rúbrica y la justicia en la evaluación. Íkala - Revista de lenguaje y cultura, v.18, n.3, p. 79-94, 2013.

LAMY, Bruno; JACQUES, Beatriz.; GALIETA, Tatiana. Funções da avaliação da aprendizagem na formação inicial de professores de Ciências. Revista Insignare Scientia $R I S$, v. 2, n. 2, p. 166-184, 19 set. 2019. DOI: https://doi.org/10.36661/2595$4520.2019 \mathrm{v} 2 \mathrm{i} 2.10851$.

MARCHETI, Ana Paula do Carmo. Rubricas: um importante instrumento para correção de desempenho discente. Revista Eletrônica Científica Ensino Interdisciplinar. Mossoró, v. 6, n. 16, p. $58-76,2020$.

MARIN, Pedronel Araque; GUTIERREZ, Sandra Adela Torijano; LONDOÑO, Nicolás Arango. Diseño e implementación de rúbricas como instrumento de evaluación del curso de Química General e inorgánica para estudiantes de Ingeniería. Rev.EIA, v. 16, n. 31, p. 131-143, jun. 2019. DOI: http://dx.doi.org/10.24050/reia.v16i31.1059.

NIEMINEN, Juuso Henrik; PESONEN, Henri Valtteri. Taking Universal Design Back to Its Roots: Perspectives on Accessibility and Identity in Undergraduate Mathematics. Education Sciences, v. 10, n. 12, 2020. DOI: 10.3390/educsci10010012.

OAKLEAF, Megan. Using rubrics to assess information literacy: an examination of methodology and interrater reliability. Journal of the Aerican Society for Information Science and Technology, v. 60, n.5, p.969-983, 2009.

RAPOSO-RIVAS, Manuela; MATÍNEZ-FIGUEIRA, Maria Esther. Evaluación educativa utilizando rúbrica: un desafío para docentes y estudiantes universitarios. Educación y Educadores, v. 17, n. 3, p. 499-513, 2014. DOI: 10.5294/edu.2014.17.3.6.

ROBLES, Daniel Cebrián; ANGULO, José Serrano; DE LA SERNA, Manuel Cebrián. Federated eRubric Service to Facilitate Self-Regulated Learning in the European University Model. European Educational Research Journal, v. 13, n. 5, p. 575-583, 2014. DOI: https://doi.org/10.2304/eerj.2014.13.5.575.

SCALLON, Gérard. L'évaluation des apprentissages dans une approche par competences. Saint-Laurent (Montréal): Éditions du Renouveau pédagagique, 2004. 
STEVENS, Dannelle; LEVI, Antonia. Introduction to Rubrics: an assessment tool to save grading time, convey effective feedback and promote student learning. Sterling, Virginia: Stylus Publishing, 2005.

TARAS, Maddalena. To feedback or not to feedback in student self-assessment. Assessment and Evaluation in Higher Education, v.28, n.5, p. 549-566, 2003.

TIERNEY, Robin; SIMON, Marielle. What's still wrong with rubrics: focusing on the consistency of performance criteria across scale levels. Practical Assessment, Research \& Evaluation, v.9, n.2, 2004.

TORMAN, Vanessa Bielefeldt Leotti; COSTER, Rodrigo; RIBOLDI, João. Normalidade de variáveis: métodos de verificação e comparação de alguns testes não-paramétricos por simulação. Clinical \& Biomedical Research, [S.1.], v. 32, n. 2, jul. 2012.

SHAPIRO, Samuel Sanford; WILK, Martin. An analysis of variance test for normality. Biometrika Trust, London, v. 52, p. 591-609, 1965.

VORPAGEL, Fernanda; UHMANN, Rosangela. Professores em Formação Discutindo a Avaliação Escolar. Revista Insignare Scientia - RIS, v. 1, n. 3, 17 fev. 2019. DOI: https://doi.org/10.36661/2595-4520.2018v1i3.10710. 